International Journal of Applied Mathematics

Volume 34 No. $6 \quad 2021,1171-1186$

ISSN: 1311-1728 (printed version); ISSN: 1314-8060 (on-line version)

doi: http://dx.doi.org/10.12732/ijam.v34i6.9

\title{
WAVELET METHODS FOR SOLVING THIRD ORDER ODEs
}

\author{
Inderdeep Singh ${ }^{1}$, Manbir Kaur ${ }^{2} \S$ \\ Department of Physical Sciences \\ Sant Baba Bhag Singh University \\ Jalandhar - 144030, INDIA
}

\begin{abstract}
In this research, we propose a numerical technique based on Hermite wavelets for solving third order ordinary differential equations, which have several applications in science and engineering. A comparative study has been presented to illustrate the accuracy of the proposed scheme. For this purpose, we have utilized Haar wavelets based approximation technique. Considerable amount of research work has been carried out to find the numerical solution of such equations, where computer symbolic systems facilitate the computational work.
\end{abstract}

AMS Subject Classification: 65N99

Key Words: Haar wavelets; Hermite wavelets; third order ODEs; operational matrices; function approximation

\section{Introduction}

Third order differential equations are arising in several applications of engineering and science. Many analytical, semi-analytical and numerical techniques have been developed for solving these differential equations such as finite difference method (FDM), Adomian decomposition method (ADM), Homotopy perturbation method (HPM), Homotopy analysis method (HAM), Runge-Kutta method (RKM) of different orders, Euler's method and Taylor's series methods. Nowadays, wavelets based approximate techniques attract the interest of scientists and researchers as wavelets based techniques are simpler, less time consuming and show very high accuracy. Basis functions of Haar wavelets have

$\begin{array}{ll}\text { Received: June 25, } 2021 & \text { (c) } 2021 \text { Academic Publications }\end{array}$

${ }^{\S}$ Correspondence author 
been used for solving differential and integral equations in [2], [3], [6], [12], [13] and [14], whereas Hermite wavelets have been used in [4], [5], [9], [10] and [11]. Some third order ordinary differential equations have been discussed in [1], [7] and $[8]$.

\section{Wavelets and their properties}

\subsection{Haar wavelets}

Haar functions are an orthogonal family of switched rectangular waveforms where amplitudes can differ from one function to another. Haar wavelet is a sequence of rescaled square shaped functions which together forms a wavelet family or basis. The Haar wavelet function $h_{i}(x)$ is defined in the interval $[\alpha, \gamma]$ as

$$
h_{i}(x)= \begin{cases}1, & \alpha \leq x<\beta, \\ -1, & \beta \leq x<\gamma, \\ 0, & \text { elsewhere }\end{cases}
$$

where $\alpha=\frac{k}{m}, \beta=\frac{k+0.5}{m}, \gamma=\frac{k+1}{m}, m=2^{j}$ and $j=0,1,2, \ldots, J . J$ denotes the level of resolution. The integer $k=0,1,2, \ldots, m-1$ is the translation parameter. The index $i$ is calculated as: $i=m+k+1$. The minimal value of $i=2$. The maximal value of $i$ is $2^{j+1}$.

The collocation points are calculated as:

$$
x_{l}=\frac{l-0.5}{2 M}, \quad l=1,2,3, \ldots ., 2 M, M=2^{J} .
$$

The operational matrix $P$, which is $2 M \times 2 M$, is calculated as below:

$$
P_{1, i}(x)=\int_{0}^{x} h_{i}(x) d x
$$

and

$$
P_{n+1, i}(x)=\int_{0}^{x} P_{n, i}(x) d x, \quad n=1,2,3, \ldots
$$

\subsection{Hermite wavelets}

Wavelets consist of a family of functions from dilation and translation of a single function known as mother wavelet. The continuous variation of dilation 
parameter $\alpha$ and translation parameter $\beta$, form a family of continuous wavelets as:

$$
\psi_{\alpha, \beta}(x)=|\alpha|^{-\frac{1}{2}} \psi\left(\frac{x-\alpha}{\beta}\right), \quad \alpha, \beta \in R, \quad \alpha \neq 0 .
$$

If the dilation and translation parameters are restricted to discrete values by setting $\alpha=\alpha_{0}{ }^{-k}, \beta=n \beta_{0} \alpha_{0}^{-k}, \alpha_{0}>1, \beta_{0}>0$, we obtain the following family of discrete wavelets:

$$
\psi_{k, n}(x)=|\alpha|^{-\frac{1}{2}} \psi\left(\alpha_{0}{ }^{k} x-n \beta_{0}\right), \quad \alpha, \beta \in R, \quad \alpha \neq 0,
$$

where $\psi_{k, n}$, form a wavelet basis for $L^{2}(R)$. For special case, if $\alpha_{0}=2$ and $\beta_{0}=1$, then $\psi_{k, n}(x)$ forms an orthonormal basis. Hermite wavelets are defined as:

$$
\psi_{n, m}(x)= \begin{cases}\frac{2^{\frac{k+1}{2}}}{\sqrt{\pi}} H_{m}\left(2^{k} m-2 n+1\right), & \frac{n-1}{2^{k-1}} \leq x \leq \frac{n}{2^{k-1}}, \\ 0, & \text { otherwise }\end{cases}
$$

where $m=0,1,2,3, \ldots, M-1$ and $n=1,2,3, \ldots, 2^{k-1}$ and $k$ is assumed any positive integer. Also, $H_{m}$ are Hermite polynomials of degree $m$ with respect to weight function $W(x)=\sqrt{1-x^{2}}$ on the real line $R$ and satisfies the following recurrence relation

$$
H_{m+2}(x)=2 x H_{m+1}(x)-2(m+1) H_{m}(x),
$$

where $m=0,1,2, \ldots, H_{0}(x)=1$ and $H_{1}(x)=2 x$.

\section{Function Approximation}

\subsection{Haar wavelets}

Consider any square integrable function $y(x)$ can be expanded in terms of infinite series of Haar basis functions as:

$$
y(x)=\sum_{i=1}^{\infty} a_{i} h_{i}(x)
$$

where $a_{i}$ are constants of this infinite series, known as Haar wavelet coefficients. For numerical approximation the above infinite series is truncated upto finite terms as:

$$
y(x)=\sum_{i=1}^{2 M} a_{i} h_{i}(x)=A^{T} h(x),
$$


where $A$ and $h(x)$ are $2 M \times 1$ matrices and are given by

$$
A^{T}=\left[a_{1}, a_{2}, \ldots, a_{2 M}\right]
$$

and

$$
h(x)=\left[h_{1}(x), h_{2}(x), \ldots, h_{2 M}(x)\right]^{T} .
$$

\subsection{Hermite wavelets}

Consider any square integrable function $u(x)$ can be expanded in terms of infinite series of Hermite basis functions as:

$$
u(x)=\sum_{n=1}^{\infty} \sum_{m=0}^{\infty} C_{n, m} \psi_{n, m}(x),
$$

where $C_{n, m}$ are constants of this infinite series, known as Hermite wavelet coefficients. For numerical approximation the above infinite series is truncated upto finite terms as:

$$
u(x)=\sum_{n=1}^{2^{k-1}} \sum_{m=0}^{M-1} C_{n, m} \psi_{n, m}(x)=C^{T} \Psi(x),
$$

where $C$ and $\Psi$ are $2^{k-1} M \times 1$ matrices and are given by

$$
C^{T}=\left[C_{1,0}, \ldots, C_{1, M-1}, \ldots, C_{2^{k-1}, 0}, \ldots, C_{2^{k-1}, M-1}\right]
$$

and

$$
\Psi=\left[\psi_{1,0}, \ldots, \psi_{1, M-1}, \ldots, \psi_{2^{k-1}, 0}, \ldots, \psi_{2^{k-1}, M-1}\right]^{T} .
$$

\section{Proposed methods for solving third order ODEs}

Consider the following third order ODE

$$
a y^{\prime \prime \prime}(x)+b y^{\prime \prime}(x)+c y^{\prime}(x)+d y(x)=F,
$$

with initial conditions $y(0)=\alpha, y^{\prime}(0)=\beta$ and $y^{\prime \prime}(0)=\gamma$, where $\alpha, \beta, \gamma$ are any constants and $a, b, c, d$ and $F$ are either functions of $x$ or constants. 


\subsection{Haar wavelet collocation method(HWCM)}

Consider the wavelet approximation

$$
y^{\prime \prime \prime}(x)=\sum_{i=1}^{2 M} a_{i} h_{i}(x)
$$

Integrating (18), thrice with respect to $x$, from 0 to $x$, we get

$$
\begin{gathered}
y^{\prime \prime}(x)=y^{\prime \prime}(0)+\sum_{i=1}^{2 M} a_{i} P_{1, i}(x), \\
y^{\prime}(x)=y^{\prime}(0)+x y^{\prime \prime}(0)+\sum_{i=1}^{2 M} a_{i} P_{2, i}(x), \\
y(x)=y(0)+x y^{\prime}(0)+\frac{x^{2}}{2} y^{\prime \prime}(0)+\sum_{i=1}^{2 M} a_{i} P_{3, i}(x) .
\end{gathered}
$$

Substituting $y^{\prime \prime \prime}, y^{\prime \prime}, y^{\prime}$ and $y$ in (17), and applying initial conditions, we get

$$
\sum_{i=1}^{2 M}\left[a h_{i}(x)+b P_{1, i}(x)+c P_{2, i}(x)+d P_{3, i}(x)\right]=G(x),
$$

where $G(x)$ contains remaining terms including $y^{\prime \prime}(0), y^{\prime}(0)$ and $y(0)$. From $(22)$, we get Haar wavelet coefficients. The Haar wavelet solution $y(x)$ is obtained by substituting the values of wavelet coefficients into (21).

\subsection{Hermite wavelet collocation method (HeWCM)}

Consider the wavelet approximation

$$
y^{\prime \prime \prime}(x)=\sum_{n=1}^{2^{k-1}} \sum_{m=0}^{M-1} C_{n, m} \psi_{n, m}(x)=C^{T} \Psi(x),
$$

Integrating (23) with respect to $x$, from 0 to $x$, we get

$$
\begin{gathered}
y^{\prime \prime}(x)=y^{\prime \prime}(0)+\sum_{n=1}^{2^{k-1}} \sum_{m=0}^{M-1} C_{n, m} \int_{0}^{x} \psi_{n, m}(x) d x, \\
y^{\prime \prime}(x)=y^{\prime \prime}(0)+\sum_{n=1}^{2^{k-1}} \sum_{m=0}^{M-1} C_{n, m} P \psi_{n, m}(x),
\end{gathered}
$$

where

$$
P \psi_{n, m}(x)=\int_{0}^{x} \psi_{n, m}(x) d x
$$


Integrating (25) with respect to $x$, we obtain

$$
\begin{gathered}
y^{\prime}(x)=y^{\prime}(0)+x y^{\prime \prime}(0)+\sum_{n=1}^{2^{k-1}} \sum_{m=0}^{M-1} C_{n, m} \int_{0}^{x} P \psi_{n, m}(x) d x, \\
y^{\prime}(x)=y^{\prime}(0)+x y^{\prime \prime}(0)+\sum_{n=1}^{2^{k-1}} \sum_{m=0}^{M-1} C_{n, m} Q \psi_{n, m}(x),
\end{gathered}
$$

where

$$
Q \psi_{n, m}(x)=\int_{0}^{x} P \psi_{n, m}(x) d x .
$$

Again, integrating (28) with respect to $x$, we obtain

$$
\begin{gathered}
y(x)=y(0)+x y^{\prime}(0)+\frac{x^{2}}{2} y^{\prime \prime}(0)+\sum_{n=1}^{2^{k-1}} \sum_{m=0}^{M-1} C_{n, m} \int_{0}^{x} Q \psi_{n, m}(x) d x, \\
y(x)=y(0)+x y^{\prime}(0)+\frac{x^{2}}{2} y^{\prime \prime}(0)+\sum_{n=1}^{2^{k-1}} \sum_{m=0}^{M-1} C_{n, m} R \psi_{n, m}(x),
\end{gathered}
$$

where

$$
R \psi_{n, m}(x)=\int_{0}^{x} Q \psi_{n, m}(x) d x .
$$

Substituting the values of $y^{\prime \prime \prime}, y^{\prime \prime}, y^{\prime}$ and $y$ in (17), and applying initial conditions, we get

$$
\sum_{n=1}^{2^{k-1} M-1} \sum_{m=0} C_{n, m}\left[a \psi_{n, m}(x)+b P \psi_{n, m}(x)+c Q \psi_{n, m}(x)+d R \psi_{n, m}(x)\right]=G_{1}(x),
$$

where $G_{1}(x)$ contains all the remaining terms. From (33), we get Hermite wavelet coefficients. The Hermite wavelet solution $y(x)$ is obtained by substituting the values of wavelet coefficients into (31).

\section{Convergence Analysis}

\subsection{Convergence of Haar wavelet collocation method}

If $y_{e}(x)$ is the exact solution and $y_{a}(x)$ is the approximate solution of the differential equation (1). Let $E_{m}$ be the corresponding error function and is defined as: 


$$
E_{m}=\left|y_{e}(x)-y_{a}(x)\right| \simeq \sum_{j=J+1}^{\infty} \sum_{k=0}^{2^{j}-1} a_{2^{j}+k} h_{2^{j}+k}(x), x \in[0,1] .
$$

Theorem. Suppose that $y(x)$ satisfies a Lipschitz's condition on $[0,1]$, there exists constant $S>0$ (dependent on both $y(x)$ and interval), such that

$$
\left|y\left(x_{1}\right)-y\left(x_{2}\right)\right| \leq S\left|x_{1}-x_{2}\right|, \quad \forall x_{1}, x_{2} \in[0,1] .
$$

Then, the Haar wavelet method will be convergent in the sense that $E_{m}$ goes to zero as $m$ goes to infinity. The order of convergence is:

$$
\left\|E_{m}\right\|_{2} \simeq O\left(\frac{1}{m}\right)
$$

Proof. Since

$$
\left\|E_{m}\right\|_{2}^{2}=\int_{0}^{1}\left(\sum_{j=J+1}^{\infty} \sum_{k=0}^{2^{j}-1} a_{2^{j}+k} h_{2^{j}+k}(x)\right)^{2} d x .
$$

After splitting the summation, from (37), we obtain

$$
\begin{aligned}
\left\|E_{m}\right\|_{2}^{2}= & \sum_{j=J+1}^{\infty} \sum_{k=0}^{2^{j}-1} a_{2^{j}+k}^{2} \int_{0}^{1} h_{2^{j}+k}^{2}(x) d x \\
& +\sum_{j=J+1}^{\infty} \sum_{k=0}^{2^{j}-1} \sum_{p=J+1}^{\infty} \sum_{q=0, q \neq k}^{2^{p}-1} a_{2^{j}+k} a_{2^{p}+q} \int_{0}^{1} h_{2^{j}+k}(x) h_{2^{p}+q}(x) d x .
\end{aligned}
$$

Using orthogonality condition, we obtain

$$
\left\|E_{m}\right\|_{2}^{2}=\sum_{j=J+1}^{\infty} \sum_{k=0}^{2^{j}-1} a_{2^{j}+k}^{2}\left(\frac{1}{2^{j}}\right) .
$$

We know that

$$
a_{2^{j}+k}=2^{j} \int_{0}^{1} y(x) h_{i}(x) d x .
$$

By the mean value theorem and equation (40), there exist

$$
x_{11}^{j k} \in\left[\frac{k}{2^{j}}, \frac{k+0.5}{2^{j}}\right], \quad x_{22}^{j k} \in\left[\frac{k+0.5}{2^{j}}, \frac{k+1}{2^{j}}\right],
$$

such that 


$$
a_{2^{j}+k}=2^{j}\left[\left(\frac{k+0.5}{2^{j}}-\frac{k}{2^{j}}\right) y\left(x_{11}^{j k}\right)-\left(\frac{k+1}{2^{j}}-\frac{k+0.5}{2^{j}}\right) y\left(x_{22}^{j k}\right)\right] .
$$

After simplification, from (42), we obtain

$$
a_{2^{j}+k}=\frac{1}{2}\left[y\left(x_{11}^{j k}\right)-y\left(x_{22}^{j k}\right)\right] .
$$

From (35) and (43), we obtain

$$
a_{2^{j}+k} \leq \frac{1}{2} S\left(x_{11}^{j k}-x_{22}^{j k}\right) \leq\left(\frac{1}{2}\right) S\left(\frac{1}{2^{j}}\right) \simeq S\left(\frac{1}{2^{j+1}}\right) .
$$

From (39) and (44), we obtain

$$
\left\|E_{m}\right\|_{2}^{2}=\sum_{j=J+1}^{\infty} \sum_{k=0}^{2^{j}-1} S^{2} \frac{1}{2^{2 j+2}}\left(\frac{1}{2^{j}}\right) .
$$

After simplification, from (45), we obtain

$$
\left\|E_{m}\right\|_{2}^{2}=\frac{S^{2}}{4} \sum_{j=J+1}^{\infty} 2^{j}\left(\frac{1}{2^{3 j}}\right) .
$$

After summation of series, from (46), we obtain

$$
\left\|E_{m}\right\|_{2}^{2}=\frac{S^{2}}{3}\left(\frac{1}{2^{J+1}}\right)^{2} .
$$

But, $m=2^{J+1}$, we obtain,

$$
\left\|E_{m}\right\|_{2} \simeq O\left(\frac{1}{m}\right) .
$$

Thus, when $m$ goes to infinity, then, $E_{m}$ tends to zero.

\subsection{Convergence of Hermite wavelet collocation method [11]}

Theorem. Consider a function $u(x)$, which is continuous and bounded in $H^{2}[0,1)$, then the Hermite wavelet expansion of $u(x)$ converge to it.

Proof. The Hermite wavelet coefficients of a continuous function $u(x)$ are defined as:

$$
C_{n, m}=\int_{0}^{1} u(x) \psi_{n, m}(x) d x .
$$

Substituting the value of $\psi_{n, m}(x)$, from (7), we obtain

$$
C_{n, m}=\int_{\Omega} u(x) \frac{2^{\frac{k+1}{2}}}{\sqrt{\pi}} H_{m}\left(2^{k} x-2 n+1\right) d x,
$$

where $\Omega=\frac{n-1}{2^{k-1}} \leq x \leq \frac{n}{2^{k-1}}$. Substituting $2^{k} x-2 n+1=s$, we obtain

$$
C_{n, m}=\frac{2^{\frac{k+1}{2}}}{\sqrt{\pi}} \int_{-1}^{1} u\left(\frac{s-1+2 n}{2^{k}}\right) H_{m}(s) 2^{-k} d s,
$$




$$
C_{n, m}=\frac{2^{\frac{-k+1}{2}}}{\sqrt{\pi}} \int_{-1}^{1} u\left(\frac{s-1+2 n}{2^{k}}\right) H_{m}(s) d s .
$$

Using GMVT for integrals, we obtain

$$
C_{n, m}=\frac{2^{\frac{-k+1}{2}}}{\sqrt{\pi}} u\left(\frac{z-1+2 n}{2^{k}}\right) \int_{-1}^{1} H_{m}(s) d s, \quad \text { for some } z \in(-1,1) .
$$

Set $\int_{-1}^{1} H_{m}(s) d s=K$, we obtain

$$
\left|C_{n, m}\right|=\left|\frac{2^{\frac{-k+1}{2}}}{\sqrt{\pi}}\right| \cdot\left|u\left(\frac{z-1+2 n}{2^{k}}\right)\right| \cdot|K| .
$$

As $u$ is bounded, therefore, $\sum_{n, m=0}^{\infty} C_{n, m}$ converges absolutely. Hence the Hermite series expansion of $u$ is convergent.

\section{Numerical Observations}

In this section, some numerical illustrations have been presented to obtain the accuracy and efficiency of these numerical schemes. Numerical results and accuracy of the proposed schemes have been obtained by using $k=1, M=8$ for Hermite wavelets collocation method (HeWCM) and $2 M=8$ for Haar wavelets collocation method (HWCM).

Example 1. Consider the third order linear ODE

$$
y^{\prime \prime \prime}(x)=3 \sin x,
$$

with initial condition $y(0)=1, y^{\prime}(0)=0$ and $y^{\prime \prime}(0)=-2$. The exact solution of the equation is

$$
y(x)=3 \cos x+\frac{x^{2}}{2}-2 .
$$

Table 1 represents the comparison of numerical solutions obtained by Haar and Hermite wavelet methods with exact solution of Example 1. Table 2 represents the comparison of absolute errors obtained by Haar wavelet method and Hermite wavelet method.

Example 2. Consider the third order ODE

$$
y^{\prime \prime \prime}(x)=-y(x),
$$

with initial condition $y(0)=1, y^{\prime}(0)=-1$ and $y^{\prime \prime}(0)=1$. The exact solution of the equation is

$$
y(x)=e^{-x} .
$$




\begin{tabular}{|c|c|c|c|}
\hline$x$ & Exact solution & $\begin{array}{c}\text { Hermite wavelet } \\
\text { solution }\end{array}$ & $\begin{array}{c}\text { Haar wavelet } \\
\text { solution }\end{array}$ \\
\hline $1 / 16$ & 0.9960956571 & 0.9960956570 & 0.9961013744 \\
\hline $3 / 16$ & 0.9649980643 & 0.9649980642 & 0.9650647394 \\
\hline $5 / 16$ & 0.9035319691 & 0.9035319691 & 0.9037200851 \\
\hline $7 / 16$ & 0.8131441752 & 0.8131441752 & 0.8135132729 \\
\hline $9 / 16$ & 0.6959766226 & 0.6959766226 & 0.6965848480 \\
\hline $11 / 16$ & 0.5548329634 & 0.5548329633 & 0.5557366367 \\
\hline $13 / 16$ & 0.3931348116 & 0.3931348115 & 0.3943880248 \\
\hline $15 / 16$ & 0.2148683502 & 0.2148683501 & 0.2165225995 \\
\hline
\end{tabular}

Table 1: Numerical solutions of Example 1.

\begin{tabular}{|c|c|c|}
\hline$x$ & $\begin{array}{c}\text { Absolute errors for } \\
\text { Haar wavelet }\end{array}$ & $\begin{array}{c}\text { Absolute errors for } \\
\text { Hermite wavelet }\end{array}$ \\
\hline $1 / 6$ & $5.7173 e-006$ & $3.7725 e-013$ \\
\hline $3 / 16$ & $6.6675 e-005$ & $4.6695 e-012$ \\
\hline $5 / 16$ & $1.8812 e-004$ & $1.3043 e-011$ \\
\hline $7 / 16$ & $3.6910 e-004$ & $2.5534 e-011$ \\
\hline $9 / 16$ & $6.0823 e-004$ & $4.2077 e-011$ \\
\hline $11 / 16$ & $9.0367 e-004$ & $6.2671 e-011$ \\
\hline $13 / 16$ & $1.2532 e-003$ & $8.7210 e-011$ \\
\hline $15 / 16$ & $1.6542 e-003$ & $1.1565 e-010$ \\
\hline
\end{tabular}

Table 2: Comparison of absolute errors of Example 1.

\begin{tabular}{|c|c|c|c|}
\hline$x$ & Exact solution & $\begin{array}{c}\text { Hermite wavelet } \\
\text { solution }\end{array}$ & $\begin{array}{c}\text { Haar wavelet } \\
\text { solution }\end{array}$ \\
\hline $1 / 16$ & 0.9394130628 & 0.9394130628 & 0.9394149001 \\
\hline $3 / 16$ & 0.8290291181 & 0.8290291181 & 0.8290505437 \\
\hline $5 / 16$ & 0.7316156289 & 0.7316156289 & 0.7316752258 \\
\hline $7 / 16$ & 0.6456485264 & 0.6456485264 & 0.6457638052 \\
\hline $9 / 16$ & 0.5697828247 & 0.5697828247 & 0.5699702485 \\
\hline $11 / 16$ & 0.5028315779 & 0.5028315780 & 0.5031065403 \\
\hline $13 / 16$ & 0.4437473100 & 0.4437473101 & 0.4441240699 \\
\hline $15 / 16$ & 0.3916056266 & 0.3916056267 & 0.3920972039 \\
\hline
\end{tabular}

Table 3: Numerical solutions of Example 2. 


\begin{tabular}{|c|c|c|}
\hline$x$ & $\begin{array}{c}\text { Absolute errors for } \\
\text { Haar wavelet }\end{array}$ & $\begin{array}{c}\text { Absolute errors for } \\
\text { Hermite wavelet }\end{array}$ \\
\hline $1 / 6$ & $1.8373 e-006$ & $1.8874 e-013$ \\
\hline $3 / 16$ & $2.1426 e-005$ & $2.3351 e-012$ \\
\hline $5 / 16$ & $5.9597 e-005$ & $6.5320 e-012$ \\
\hline $7 / 16$ & $1.1528 e-004$ & $1.2779 e-011$ \\
\hline $9 / 16$ & $1.8742 e-004$ & $2.1016 e-011$ \\
\hline $11 / 16$ & $2.7496 e-004$ & $3.1186 e-011$ \\
\hline $13 / 16$ & $3.7676 e-004$ & $4.3160 e-011$ \\
\hline $15 / 16$ & $4.9158 e-004$ & $5.6794 e-011$ \\
\hline
\end{tabular}

Table 4: Comparison of absolute errors of Example 2.

Table 3 represents the comparison of numerical solutions obtained by Haar and Hermite wavelet methods with exact solution of Example 2. Table 4 represents the comparison of absolute errors obtained by Haar wavelet method and Hermite wavelet method.

Example 3. Consider the following third order ODE

$$
y^{\prime \prime \prime}(x)+4 y^{\prime}(x)=x,
$$

with initial condition $y(0)=0, y^{\prime}(0)=0$ and $y^{\prime \prime}(0)=1$. The exact solution of the equation is

$$
y(x)=\frac{3}{16}(1-\cos 2 x)+\frac{x^{2}}{8} .
$$

\begin{tabular}{|c|c|c|c|}
\hline$y$ & Exact solution & $\begin{array}{c}\text { Hermite wavelet } \\
\text { solution }\end{array}$ & $\begin{array}{c}\text { Haar wavelet } \\
\text { solution }\end{array}$ \\
\hline $1 / 6$ & 0.0019512186 & 0.0019512187 & 0.0019455547 \\
\hline $3 / 16$ & 0.0174243521 & 0.0174243531 & 0.0173590571 \\
\hline $5 / 16$ & 0.0476514463 & 0.0476514492 & 0.0474724339 \\
\hline $7 / 16$ & 0.0912388703 & 0.0912388758 & 0.0909026849 \\
\hline $9 / 16$ & 0.1462051843 & 0.1462051930 & 0.1456832055 \\
\hline $11 / 16$ & 0.2101043360 & 0.2101043482 & 0.2093857463 \\
\hline $13 / 16$ & 0.2801777440 & 0.2801777600 & 0.2792710024 \\
\hline $15 / 16$ & 0.3535258136 & 0.3535258333 & 0.3524584928 \\
\hline
\end{tabular}

Table 5: Numerical solutions of Example 3. 


\begin{tabular}{|c|c|c|}
\hline$y$ & $\begin{array}{c}\text { Absolute errors for } \\
\text { Haar wavelet }\end{array}$ & $\begin{array}{c}\text { Absolute errors for } \\
\text { Hermite wavelet }\end{array}$ \\
\hline $1 / 6$ & $5.6639 e-006$ & $8.6019 e-011$ \\
\hline $3 / 16$ & $6.5295 e-005$ & $1.0541 e-009$ \\
\hline $5 / 16$ & $1.7901 e-004$ & $2.8850 e-009$ \\
\hline $7 / 16$ & $3.3619 e-004$ & $5.4749 e-009$ \\
\hline $9 / 16$ & $5.2198 e-004$ & $8.6529 e-009$ \\
\hline $11 / 16$ & $7.1859 e-004$ & $1.2230 e-008$ \\
\hline $13 / 16$ & $9.0674 e-004$ & $1.5975 e-008$ \\
\hline $15 / 16$ & $1.0673 e-003$ & $1.9663 e-008$ \\
\hline
\end{tabular}

Table 6: Comparison of absolute errors of Example 3.

Table 5 represents the comparison of numerical solutions obtained by Haar and Hermite wavelet methods with exact solution of Example 3. Table 6 represents the comparison of absolute errors obtained by Haar wavelet method and Hermite wavelet method.

Example 4. Consider the following third order ODE

$$
y^{\prime \prime \prime}(x)=e^{-x}, \quad 0 \leq x \leq 1
$$

with initial condition $y(0)=3, y^{\prime}(0)=1$ and $y^{\prime \prime}(0)=5$. The exact solution of the equation is

$$
y(x)=2+2 x^{2}+e^{x} .
$$

Figure 1 and Figure 2 show the absolute errors obtained by Hermite wavelets and Haar wavelets based collocation methods respectively for Example 4.

Example 5. Consider the following third order ODE

$$
y^{\prime \prime \prime}(x)=y^{\prime \prime}(x)-y^{\prime}(x)+y(x)+e^{-x}, \quad 0 \leq x \leq 1
$$

with initial condition $y(0)=1, y^{\prime}(0)=1$ and $y^{\prime \prime}(0)=0$. The exact solution of the equation is

$$
y(x)=\frac{1}{2} x e^{x}+\cos x+\frac{1}{2} \sin x .
$$

Figure 3 and Figure 4 show the absolute errors obtained by Hermite wavelets and Haar wavelets based collocation methods respectively for Example 5. 


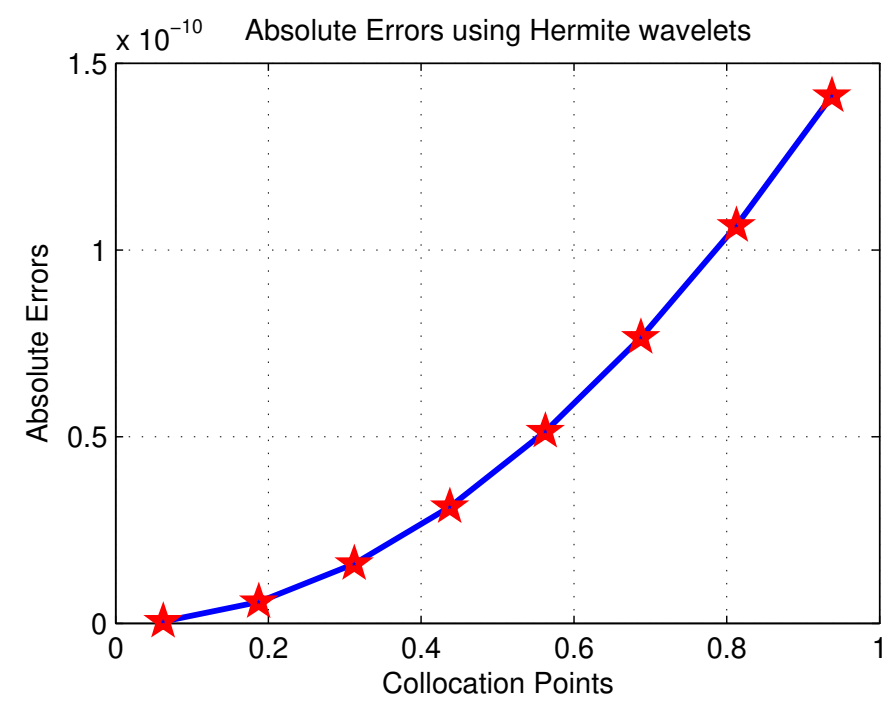

Figure 1: Absolute errors using Hermite wavelets for Example 4

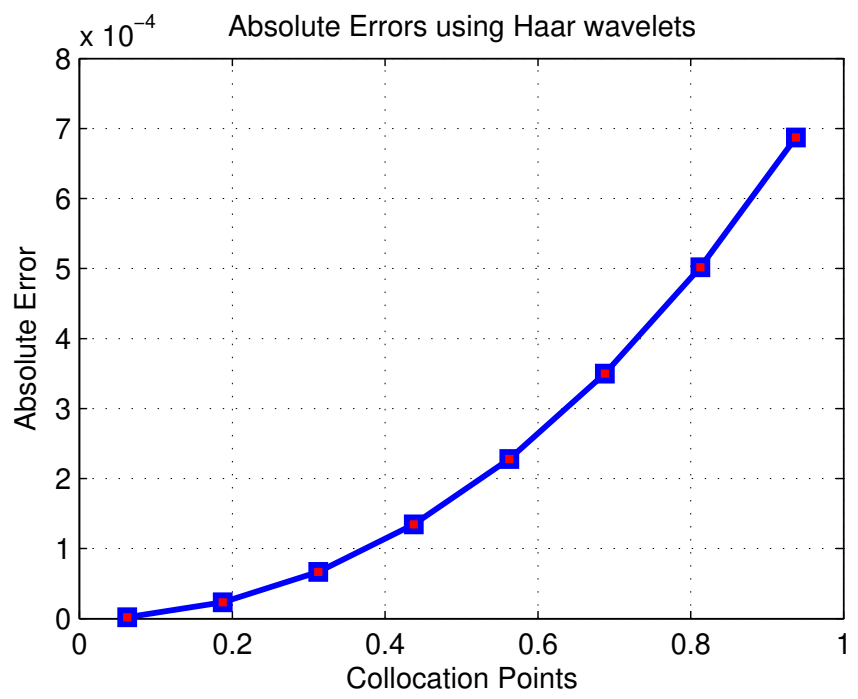

Figure 2: Absolute errors using Haar wavelets for Example 4 


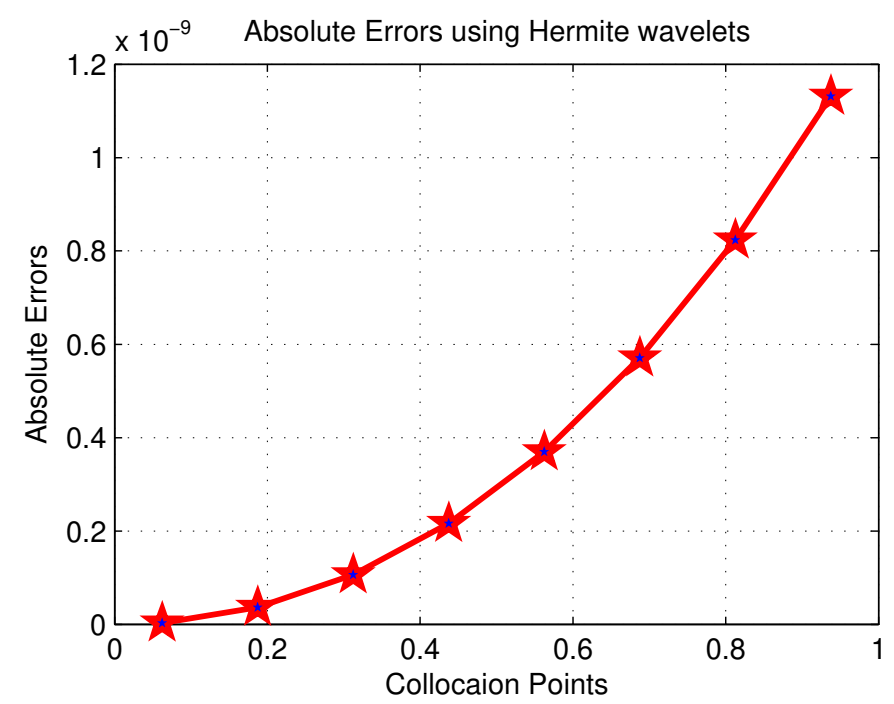

Figure 3: Absolute errors using Hermite wavelets for Example 5

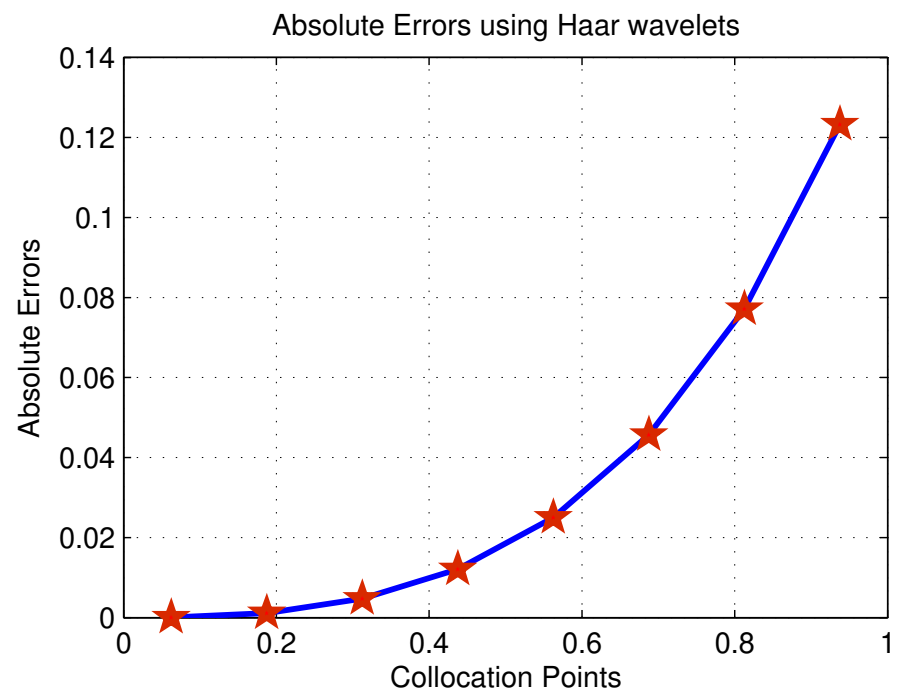

Figure 4: Absolute errors using Haar wavelets for Example 5 


\section{Conclusion}

From above discussion, it is concluded that the Hermite wavelet based collocation method is much better in comparison to Haar wavelet based collocation method for solving third order ordinary differential equations arising in science and engineering. To obtain more accurate results, the number of collocation points may be increased.

\section{Acknowledgement}

We are thankful to Editor and Reviewers for their valuable suggestions to improve the paper in its present form.

\section{References}

[1] T.A. Anake, A.O. Adesanya, G.J. Oghonyon and M.C. Agarana, Block algorithm for general third order ordinary differential equation, ICASTOR J. of Math. Sciences, 7, No 2 (2013), 127-136.

[2] C. Cattani, Haar wavelet splines, J. Interdisciplinary Math., 4 (2001), 3547.

[3] C.F. Chen, C.H. Hsiao, Wavelet approach to optimising dynamic systems, IEEE Proc. Control Theory and Appl., 146 (1997), 213-219.

[4] M. Kaur, I. Singh, Hermite wavelet method for solving oscillatory electrical circuit equations, J. Math. Comput. Sci., 11, No 5 (2021), 6266-6278.

[5] B.I. Khashem, Hermite wavelet approach to estimate solution for Bratu's problem, Emirates J. for Engin. Research, 24, No 2 (2019), 1-4.

[6] Ü. Lepik, Numerical solution of differential equations using Haar wavelets, Mathematics and Computers in Simulation, 68 (2005), 127-143.

[7] Z.A. Majid, N.A. Azmi, M. Suleman and Z.B. Ibrahaim, Solving direct general third order ordinary differential equations using two-point four step block method, Sains Malaysiana, 41, No 5 (2012), 623-632.

[8] Z. Omar, J.O. Kuboye, Computation of an accurate implicit block method for solving third order ordinary differential equations directly, Global J. of Pure and Appl. Math., 11, No 1 (2015), 177-186. 
[9] O. Oruc, A numerical procedure based on Hermite wavelets for two dimensional hyperbolic telegraph equation, Engineering with Computers, 34, No 4 (2018), 741-755.

[10] N.A. Pirim, F. Ayaz, Hermite collocation method for fractional order differential equations, An Intern. J. of Optimization and Control: Theories \& Applications, 8, No 2 (2018), 228-236.

[11] S.C. Shiralashetti, K. Srinivasa, Hermite wavelets operational matrix of integration for the numerical solution of nonlinear singular initial value problems, Alexandria Engin. J., 57 (2018), 2591-2600.

[12] I. Singh, Wavelet based method for solving generalized Burger's type equations, Intern. J. of Computational Materials Science and Engineering, 8, No 4 (2019), 1-24.

[13] I. Singh, S. Kumar, Haar wavelet method for some nonlinear Volterra integral equations of the first kind, J. of Computational and Applied Mathematics, 292 (2016), 541-552.

[14] I. Singh, S. Kumar, Haar wavelet collocation method for solving nonlinear Kuramoto-Sivashinsky equation, Italian J. of Pure and Appl. Math., 39 (2018), 373-384. 